\title{
Bone Regeneration Using Collagenated Synthetic Bone Graft Attached to Collagen Membrane with 3,4-dihydroxy-L-phenylalanine (DOPA)
}

\section{OPEN ACCESS}

pISSN 1229-5418

eISSN 2671-6623

Implantology 2021; 25(1): 10-21

https://doi.org/10.32542/implantology.2021002

Received: November 3, 2020

Revised: December 24, 2020

Accepted: January 18, 2021

\section{ORCID}

Inpyo Hong

https://orcid.org/0000-0002-0486-9593

Young Woo Song

https://orcid.org/0000-0003-1835-5646

Jae-Kook Cha

https://orcid.org/0000-0002-6906-7209

Jung-Seok Lee

https://orcid.org/0000-0003-1276-5978

Jeong-Won Paik

https://orcid.org/0000-0002-5554-8503

Seong-Ho Choi

https://orcid.org/0000-0001-6704-6124

Copyright $\odot$ 2021. The Korean Academy of Oral \& Maxillofacial Implantology

This is an Open Access article distributed under the terms of the Creative Commons Attribution Non-Commercial License (http://creativecommons. org/licenses/by-nc/4.0/) which permits unrestricted non-commercial use, distribution, and reproduction in any medium, provided the original work is properly cited.
Inpyo Hong, DDS ${ }^{1+}$, Renchindorj Dolgorsuren, DDS ${ }^{2+}$, Su-Hee Jeon, DDS ${ }^{1}$, Young Woo Song, DDS, PhD ${ }^{3}$, Jae-Kook Cha, DDS, PhD ${ }^{4}$, Jung-Seok Lee, DDS, PhD ${ }^{5}$, Jeong-Won Paik, DDS, PhD , Seong-Ho Choi, DDS, PhD ${ }^{7 *}$

${ }^{1}$ Resident, Department of Periodontology, Research Institute of Periodontal Regeneration, Yonsei University College of Dentistry, Seoul, Korea

${ }^{2}$ Postgraduate Student, Department of Periodontology, Research Institute of Periodontal Regeneration, Yonsei University College of Dentistry, Seoul, Korea

${ }^{3}$ Clinical Research Assistant Professor, Department of Periodontology, Research Institute of Periodontal Regeneration, Yonsei University College of Dentistry, Seoul, Korea

${ }^{4}$ Assistant Professor, Department of Periodontology, Research Institute of Periodontal Regeneration, Yonsei University College of Dentistry, Seoul, Korea

${ }^{5}$ Associate Professor, Department of Periodontology, Research Institute of Periodontal Regeneration, Yonsei University College of Dentistry, Seoul, Korea

${ }^{6}$ Clinical Assistant Professor, Department of Periodontology, Research Institute of Periodontal Regeneration, Yonsei University College of Dentistry, Seoul, Korea

${ }^{7}$ Professor, Department of Periodontology, Research Institute of Periodontal Regeneration, Yonsei University College of Dentistry, Seoul, Korea

${ }^{\dagger}$ Theses two authors contributed equally to this work as the co-first authors.

*Corresponding author: Seong-Ho Choi, Department of Periodontology, Research Institute for Periodontal Regeneration, Yonsei University College of Dentistry, 50 Yonsei-ro, Seodaemun-gu, Seoul 03722, Korea. Tel: +82-2-2228-3185. Fax: +82-2-392-0398. E-mail: shchoi726@yuhs.ac

\begin{abstract}
Purpose: The stability of graft material and membrane is crucial for successful guided bone regeneration. As 3,4-dihydroxy-L-phenylalanine (DOPA) is known to contribute to the adhesiveness of natural mussel adhesive proteins, the aim of this study was to evaluate the biocompatibility and bone regeneration using collagenated synthetic bone graft attached to the collagen membrane with DOPA in the rabbit calvaria model.

Materials and Methods: Four circular bone defects of $8 \mathrm{~mm}$ diameter were created in the rabbit calvarium. Each defect in the calvarium was randomly allocated to 1) sham control group, 2) bone graft using a particle-type synthetic bone substitute (OP) group, 3) bone graft using collagenated synthetic bone block (OC) group, or 4) bone graft using the collagenated synthetic bone graft attached to collagen membrane with DOPA (OC-DOPA) group. A total of 10 rabbits were sacrificed at either 2 or 8 weeks post-surgery.

Results: During histometric analysis, new bone formation in the OC-DOPA group was significantly greater than that in sham control at 8 weeks. On the contrary, there was no statistically significant difference in new bone area (NBA) between OC-DOPA and OC. Histologic analysis revealed infiltrations of inflammatory cells in the OP, OC, and OC-DOPA groups at 2 weeks. At 8 weeks, there
\end{abstract}


were no remarkable inflammatory cells in the OP and OC groups. However, multinucleated giant cells (MNGC) were observed till 8 weeks in the OC-DOPA group.

Conclusion: The collagenated synthetic bone graft attached to the collagen membrane using DOPA showed its potential for guided bone regeneration. However, MNGC were observed until the late healing period.

Keywords: Biphasic calcium phosphate, Collagen, 4-dihydroxy-L-phenylalanine, Guided bone regeneration, Multinucleated giant cell

\section{I . Introduction}

With the support of well-documented studies that showed a high success rate for guided bone regeneration (GBR), GBR is now a common procedure in implant surgery. ${ }^{1}$ Among the various surgical approaches of GBR, the application of particulate graft material and resorbable collagen membrane is the most frequently used in clinical situations with successful outcomes. ${ }^{2}$ However, it has a clinical limitation in that the graft material might get scattered and lose its augmented form during surgery and in the subsequent healing period. Since the stability of the grafted site is one of the main factors for successful GBR, scattering of bone graft and dislocation of barrier membrane or bone graft hampers bone regeneration at the surgical site. ${ }^{3}$

The addition of collagen to particulate bone graft facilitates its molding in desirable form for clinical manipulation. ${ }^{4}$ In case of uncontained defects, the formability of the collagenated bone graft offers additional stability by molding the graft material to adapt to the defect. In addition, it has the advantage of being simple in application of bone grafts during surgical procedures. With these advantages, a previous study reported successful outcomes for GBR using a collagenated synthetic bone graft in a canine model. ${ }^{5}$

For the stability of the barrier membrane, titanium pin has been used commonly for the fixation of the membrane to the GBR site. Using a titanium pin for membrane fixation provided successful bone regeneration, however, there were limitations in that the second surgery for pin removal was necessary and excessive flap elevation may be required for accessibility of titanium pins. ${ }^{6}$ For membrane fixation avoiding additional surgery for pin removal, loading mussel adhesive protein (MAP) to the membrane was suggested. ${ }^{7}$ MAP is secreted by the mussel foot of Mytilus genus and plays a crucial role in attaching to rocks in marine environments. ${ }^{8}$ A previous study using mussel adhesive protein for membrane fixation showed biocompatible and efficient outcomes for GBR in the rabbit calvaria model. ${ }^{7}$ Among the composition of mussel foot protein, 3,4-dihydroxy-L-phenylalanine (DOPA), which is a hydroxylated form of tyrosine, is known to play a crucial role in adhesive property. ${ }^{8}$ 
Thus, the combined use of collagenated synthetic bone graft and DOPA-loaded collagen membrane is expected to maximize the stability of grafted sites while avoiding an additional surgical approach. Previous studies evaluated the biocompatibility and bone regeneration of collagenated synthetic bone graft and mussel foot protein-loaded collagen membrane, respectively., ${ }^{5,7}$ However, there was no evaluation of combined use of collagenated synthetic bone graft and DOPA-loaded collagen membrane. Therefore, the aim of our study was to evaluate the biocompatibility and bone regeneration of collagenated synthetic bone graft attached to a collagen membrane using DOPA in a rabbit calvaria model.

\section{II . Materials and Methods}

\section{Animals}

Ten male New Zealand white rabbits (body weight, $2.8-3.2 \mathrm{~kg}$ ) were used. All animals were housed in standard housing conditions according to the Association for Assessment and Accreditation of Laboratory Animal Care International (AAALAC). All surgical procedures and animal management protocols were approved by the Institution Animal Care and Use Committee, Yonsei Medical Center, Seoul, Korea (approval number 2019-0123).

\section{Materials}

Collagenated synthetic bone block was used (Osteon 3 Collagen ${ }^{\circledR}$, Genoss, South Korea; [OC]). OC is a mixture of biphasic calcium phosphate (BCP), which consists of $60 \%$ hydroxyapatite (HA) and $40 \%$ $\beta$-tricalciumphosphate (TCP), and highly purified type I collagen derived from porcine tendon. It was trimmed to a round disc with a diameter of $8 \mathrm{~mm}$ and thickness of $2.5 \mathrm{~mm}$. In addition, a particle-type synthetic bone substitute was used (Osteon $3{ }^{\circledR}$, Genoss, [OP]). OP is a mixture of BCP, consisting of $60 \% \mathrm{HA}$ and $40 \% \beta-\mathrm{TCP}$.

3,4-Dihydroxy-DL-phenylalanine (DOPA) was purchased commercially(DOPA, Sigma-Aldrich Co., USA) and used as an adhesive for attaching the collagen membrane to OC.

A resorbable collagen membrane was used (Collagen Membrane- ${ }^{\circledR}{ }^{\circledR}$, Genoss, $\left.[\mathrm{CM}]\right)$. The $\mathrm{CM}$ was chemically crosslinked with type I collagen membrane derived from porcine tendon.

The collagenated synthetic bone graft attached to the collagen membrane using DOPA (OC-DOPA) was prepared using CM and OC. Just before the graft procedure, DOPA was spread on the tissue side of the trimmed CM. Afterwards, OC was applied over the DOPA-spread surface of the CM for adhesion. 


\section{Study design}

Four circular defects with a diameter of $8 \mathrm{~mm}$ were created on the rabbit calvarium with a trephine bur (Fig. 1). Each defect was randomly assigned to one of the following groups:

1. Sham control

2. Bone graft using particle type synthetic bone substitute (OP group)

3. Bone graft using collagenated synthetic bone block (OC group)

4. Bone graft using collagenated synthetic bone graft attached to the collagen membrane using DOPA (OC-DOPA group)

10 Rabbits were sacrificed at either 2 weeks $(n=5)$ or 8 weeks $(n=5)$.

\section{Surgical procedure}

The surgical procedure was described in a previous study. ${ }^{9}$ All procedures were performed under general anesthesia with alfaxan $(5 \mathrm{mg} / \mathrm{kg})$ injection and isoflurane $(2-2.5 \%)$ inhalation. Povidoneiodine was used for disinfection of the surgical site. After disinfection, 2\% lidocaine with 1:100,000 epinephrine injection was administered for local anesthesia. A full-thickness flap was elevated and four round defects of $8 \mathrm{~mm}$ diameter were created on the exposed calvarium with a trephine bur. Copious irrigation with saline was performed during drilling to prevent overheating, and intact dura mater or cerebral tissue was confirmed after drilling. Each defect was randomly allocated in the study design. After bone grafting according to the study design, flaps were carefully closed with absorbable 4-0 suture material (Monosyn, B.Braun Surgical, S.A, Rubi, Spain). General antibiotic (enfloxacin; $10 \mathrm{mg}$ /
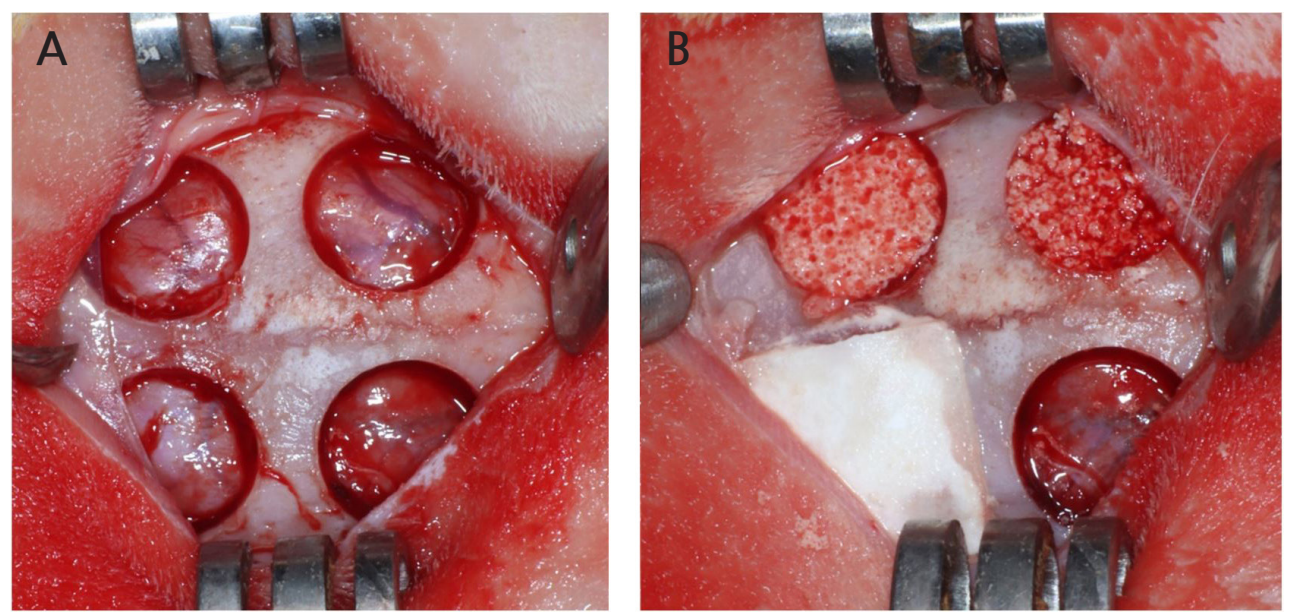

Fig. 1. Experimental design in the rabbit calvarium. (A) Circular defects $8 \mathrm{~mm}$ in diameter were prepared. (B) Each defect was randomly assigned to study group. Clockwise from top left; OC, OP, sham control, and OC-DOPA. 
day) was administered for 5 days after the operation. Each rabbit was euthanized at 2 or 8 weeks postoperatively, as designed. The obtained specimens were fixed in $10 \%$ buffered formalin for 10 days.

\section{Evaluation methods}

\section{Clinical observations}

With daily care of rabbits, clinical observation of the surgical site was performed. Abnormal inflammatory signs and unexpected complications were evaluated until sacrifice.

\section{Histologic analysis}

Histologic analysis was performed according to the method described in a previous study by Hong et al. ${ }^{9}$ The specimens were decalcified in $5 \%$ formic acid for 14 days, and $5 \mu \mathrm{m}$ thick paraffin sections were made. Sections were subsequently stained with hematoxylin-eosin and Masson's trichrome. Histologic slides were digitally captured with a light microscope (DM LB, Leica Microsystems, Germany) equipped with a camera (DC300F, Leica Microsystems). After digitalization, a computer-aided slide image analysis program (Case Viewer 2.2; 3DHISTECH Ltd., Budapest, Hungary) was used for histometric analysis of the slides. The region of interest was selected for the new bone area (NBA) measurement. The lateral margins of the regions of interest (ROIs) were defined by the lateral defect cuts made by the trephine bur. The superior border of the ROI was defined by the periosteum or the lower border of the observable collagen membrane. The inferior border of the ROI was defined by the dura mater.

\section{Statistical analysis}

Commercially available statistical software (SPSS version 23, IBM Corp., USA) was for statistical analysis. NBA measurements are summarized as median and interquartile range. Statistically significant differences between study groups were analyzed using the Kruskal-Wallis test and Mann-Whitney U test. $p$ values $<.05$ indicated statistically significant difference.

\section{Results}

\section{Clinical observations}

Surgical wounds normally healed without unexpected adverse effect. After sacrifice, normal periosteum and adjacent tissue were observed in the specimens. 
Table 1. Median [IQR] values of NBA measured by histometric analysis. Values are presented as median[IQR] $\left(\mathrm{mm}^{2}\right)$

\begin{tabular}{ccc|}
\hline Patients $(\mathrm{n}=25)$ & Demographic results & NBA $\left(\mathrm{mm}^{2}\right)$. \\
\hline \multirow{2}{*}{$\begin{array}{c}\text { weeks } \\
(\mathrm{n}=5)\end{array}$} & Sham control & $0.15[0.12-0.16]$ \\
& OP & $1.98[0.26-2.12]$ \\
& OC & $1.22[0.66-1.41]$ \\
& OC-DOPA & $1.20[0.78-1.62]$ \\
8 weeks & Sham control & $2.07[1.82-2.62]^{*}$ \\
$(\mathrm{n}=5)$ & OP & $2.66[2.64-2.68]^{*}$ \\
& OC & $4.63[3.56-4.75]^{*}$ \\
& OC-DOPA & $3.91[3.84-5.31]^{*+}$
\end{tabular}

\footnotetext{
* Statistically significant difference compared to the corresponding groups at 2 weeks.

Statistically significant difference compared to the control and OC-DOPA groups at 8 weeks

IQR: interquartile range, NBA: new bone area, OP: bone graft using particle type synthetic bone substitute, OC: bone graft using collagenated synthetic bone block, OC-DOPA: bone graft using the collagenated synthetic bone graft attached collagen membrane using DOPA.
}

\section{Histometric findings}

Histometric findings are summarized in Table 1. There was no significant difference in the NBA of all groups at 2 weeks. At 8 weeks, the NBA of OC-DOPA was significantly larger than that of OP and sham control. However, there was no statistically significant difference between the NBA of OC-DOPA and OC. Except for OC-DOPA, there was no significant difference between the NBA of OC, OP, and sham control.

\section{Histologic findings}

Figure 2 is a representative histologic image from the sham control group. At 2 weeks, the least amount of inflammatory cell was found in sham control, compared to the other study groups. Since there was no graft material, the defect space was mostly occupied with cerebral tissue infiltrating from the lower border of the defect. A small amount of new bone formation was found in the lateral border of the defect. At 8 weeks, a thin mature bone bridge was found in the defect without notable inflammation in the control group.

Figure 3 is a representative histologic image from the OC group. At 2 weeks, new bone was found in the lateral border of the defect in the OC group. In contrast, infiltration of inflammatory cells was found in the middle of the defect, which was filled with OC. Round-shaped BCP particles were observed. At 8 weeks, new bone was found around the BCP particles of OC. From the periphery, BCP particles were replaced with new bone. Invasion of the capillary was found inside the ossified BCP particles. There was no remarkable inflammatory cell infiltration throughout the defect. 

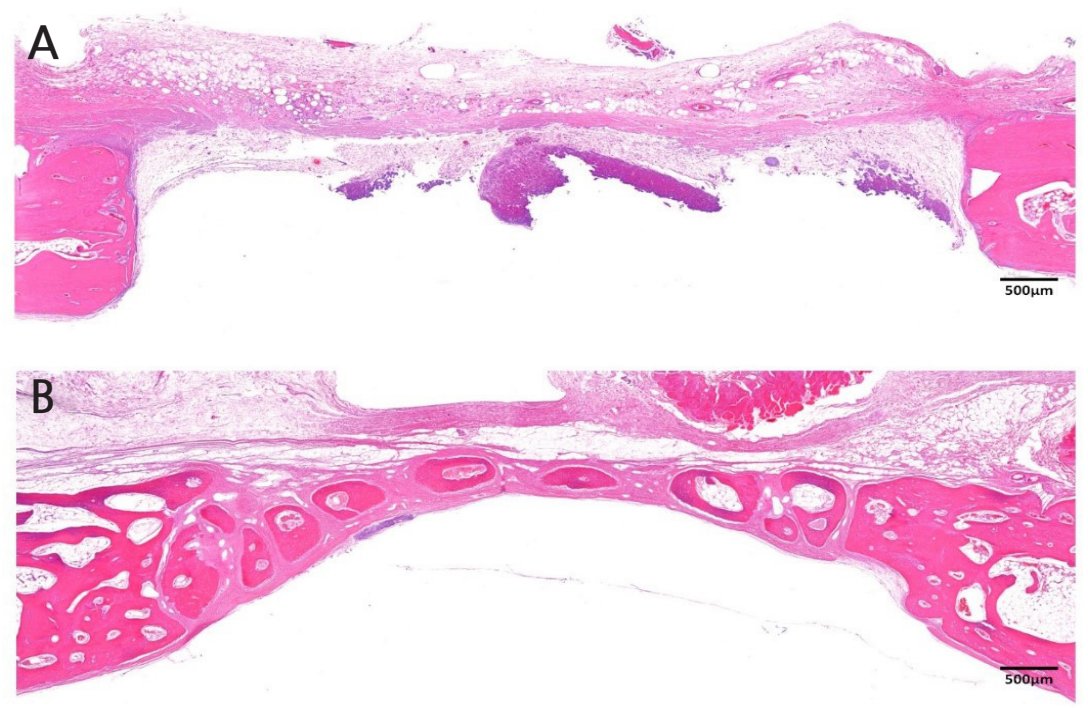

Fig. 2. Histologic image of sham control group (Hematoxylin-eosin staining). (A) Image of sham control at 2 weeks. Small amount of new bone is found in the lateral border of the defect. (B) Image of sham control at 8 weeks.
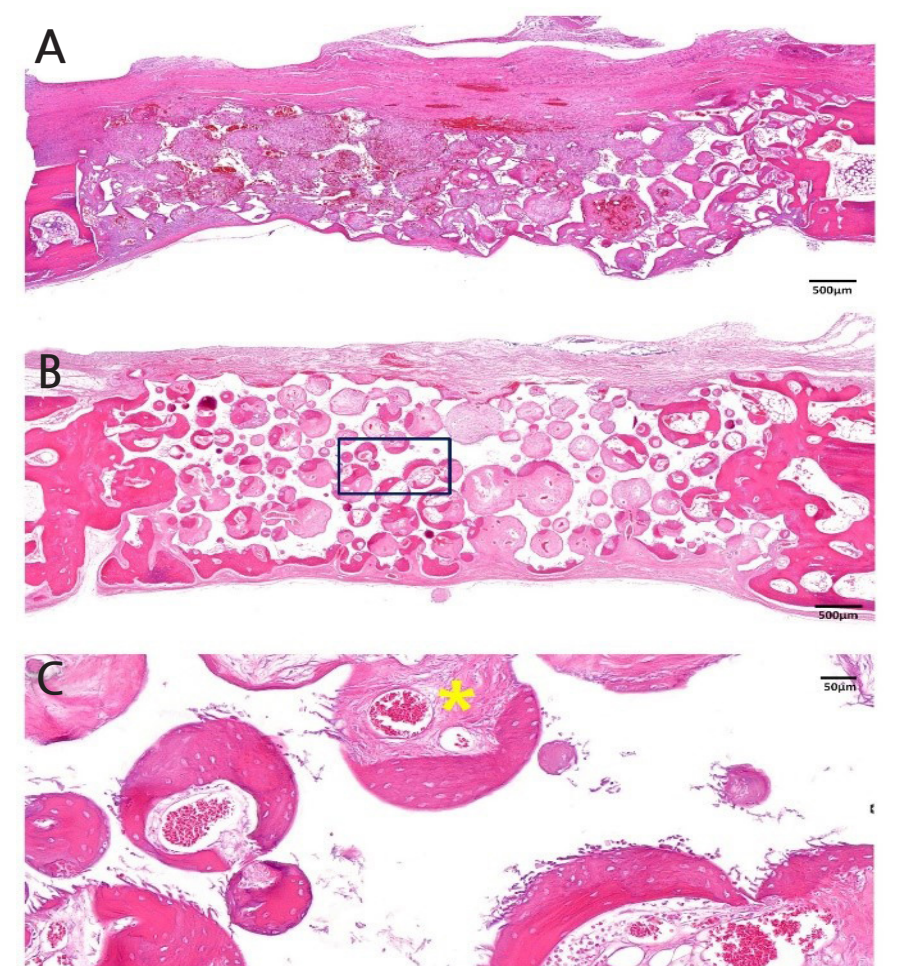

Fig. 3. Histologic image of OC group (Hematoxylin-eosin staining). (A) Image of OC group at 2 weeks. Small amount of new bone is found at the lateral border of the defect. Infiltrations of inflammatory cells were found in the middle of defect. (B) Image of OC group at 8 weeks. New bone formation was observed around BCP particles. (C) High magnification image of OC group at 8 weeks (Black squared area). Invasion of capillary (Yellow star) was found inside of the ossified BCP particles.

Magnification of the image is presented as a bar, BCP: biphasic calcium phosphate OC: Bone graft using collagenated synthetic bone block 
Figure 4 is a representative histologic image from the OP group. At 2 weeks, new bone was found in the lateral border of the defect, and infiltration of inflammatory cells was found in the middle of the defect, as the $\mathrm{OC}$ group at 2 weeks showed. Compared to OC, BCP particles in OP were sharper than those embedded in the OC block. At 8 weeks, in the OP group, BCP particles were partially replaced with new bone. The distribution of BCP was more irregular compared to that in the OC group at 8 weeks.

Figure 5 is a representative histologic image from the OC-DOPA group. At 2 weeks, new bone formation was observed from the lateral border of the defect in the OC-DOPA group. Infiltration of inflammation was found underneath the collagen membrane, where DOPA was applied. In addition, foreign body reaction and angiogenesis were observed underneath the membrane. Until 8 weeks, multinucleated giant cells (MNGC) were observed underneath the membrane. New bone formation was found at the lateral margin of the defect, and BCP particles were partially replaced with new bone.
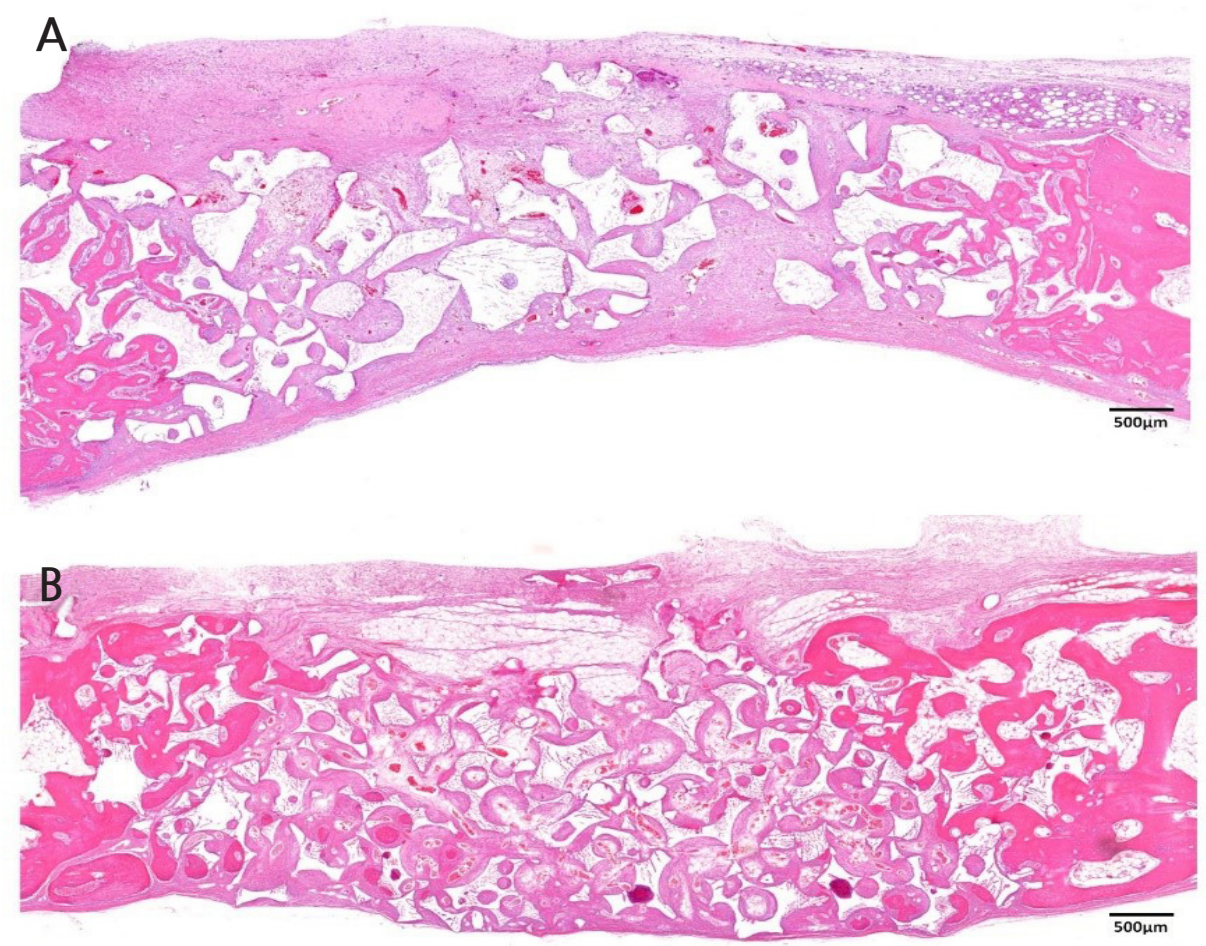

Fig. 4. Histologic image of OP group (Hematoxylin-eosin staining). (A) Image of OP group at 2 weeks. Small amount of new bone is found in the lateral border of the defect. BCP particles are more irregular compared to OC group. (B) Image of OP group at 8 weeks. BCP particles were partially replaced with new bone.

Magnification of the image is presented as a bar, BCP: biphasic calcium phosphate, OP: Bone graft using a particle-type synthetic bone substitute. 

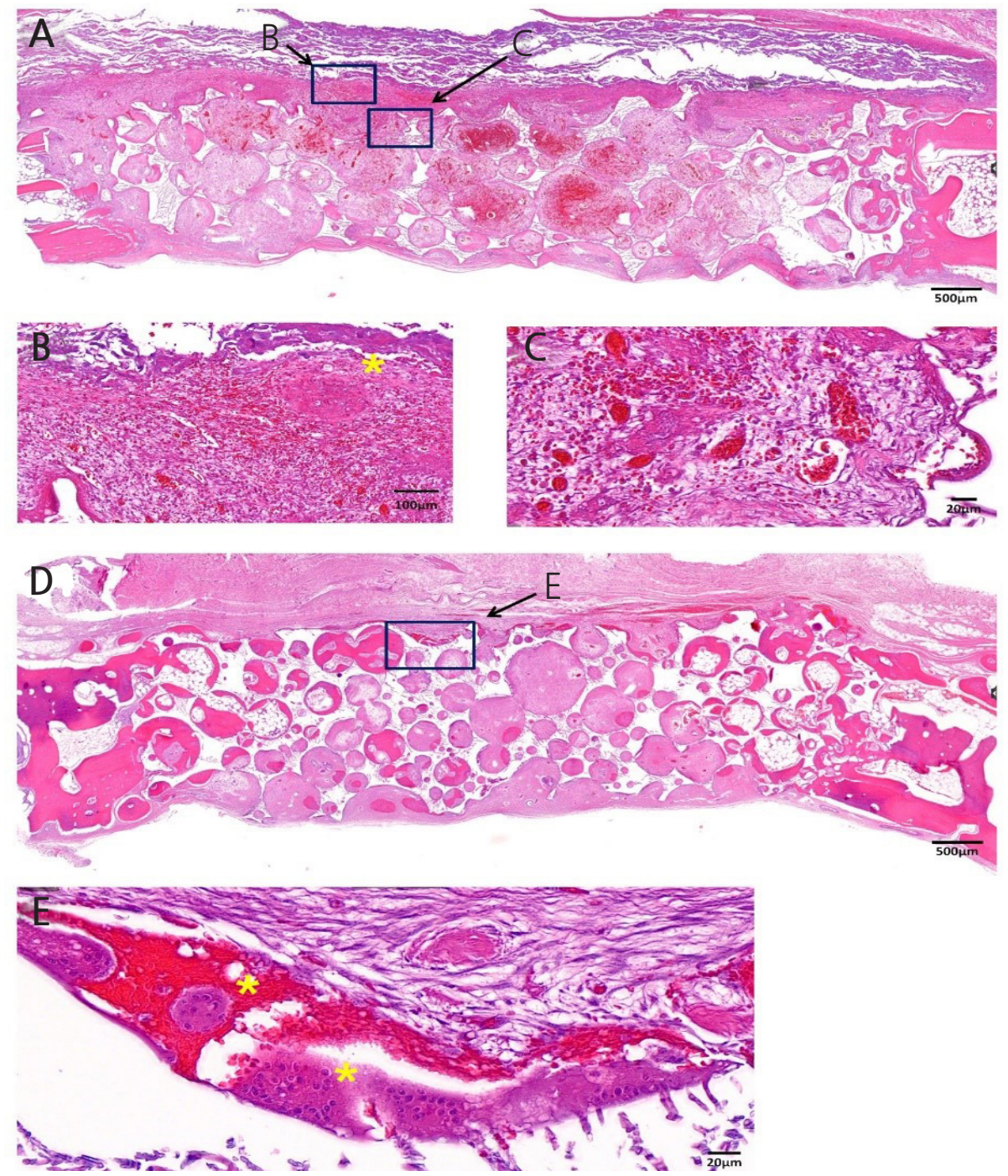

Fig. 5. Histologic image of OC-DOPA group. (A) Low magnification image of OC-DOPA group at 2 weeks. (B, C) High magnification image of OC-DOPA group at 2 weeks. (B) Infiltration of inflammatory cells are observed underneath collagen membrane. MNGC $(*)$ are seen. (C) Newly formed capillaries are observed between the membrane and the graft material. (D) Low magnification image of OCDOPA group at 8 weeks. BCP particles were partially replaced with new bone from lateral border of the defect. (E) High magnification image of OC-DOPA group at 8 weeks. MNGC $(*)$ are observed where the membrane was located.

OC-DOPA: Bone graft using the collagenated synthetic bone graft attached to the collagen membrane using DOPA, BCP: biphasic calcium phosphate, MNGC: Multinucleated giant cells. Magnification of the image is presented as a bar.

\section{Discussion}

The collagenated synthetic bone graft attached to the collagen membrane using DOPA showed proper bone regeneration in the rabbit calvaria defect model. Although DOPA-loaded collagen membranes did not show significant clinical adverse reactions or deteriorated bone regeneration, multinucleated cells 
were observed until 8 weeks of healing at DOPA application sites. Applying DOPA to the collagen membrane is expected to increase the stability of the membrane, which results in increased bone regeneration. However, at the same time, applying DOPA may induce foreign body reactions or additional inflammation.

The particulate type of bone graft, which is the most frequently used in clinical situations, has the limitation that it may collapse due to movement and the compressive force of flap closure and the healing process. ${ }^{10}$ A previous study reported that almost $40 \%$ of particulate grafts were dislocated during woundclosure procedure, which increases the possibility of dehiscence around implants placed simultaneously with GBR. ${ }^{10}$ To overcome these drawbacks, methods such as using titanium pin for fixation combined with overaugmentation of particulate bone graft or using collagenated bone block showed improvement in volume stability with predictable bone regeneration. ${ }^{10,11}$

The necessity of second surgery for pin removal and limitations in accessibility are limitations regarding the clinical application of pins during GBR. A novel method of attaching a collagen membrane and block type bone graft with MAP has been suggested for overcoming the clinical limitations of pin application. ${ }^{7}$ The authors evaluated the bone regeneration by attaching crosslinked collagen membrane and polycaprolactone (PCL) block with MAP. The results of this study confirmed higher biocompatibility of MAP, compared to cyanoacrylate, which is a chemically synthesized bio-adhesive. However, bone regeneration capacity of PCL block with collagen membrane adhered using MAP declined compared to that of the defect that was covered with only MAP spread collagen membrane without graft material.

In the previous study, PCL demonstrated the advantage of incorporating and delivering growth factors, such as bone morphogenic protein, while working as a scaffold for bone regeneration ${ }^{12}$ However, since PCL has hydrophobic characteristics, it showed a lack of new bone formation compared to conventional graft material, such as BCP or xenograft. ${ }^{13}$ In the present study, the collagenated BCP block showed bone regeneration comparable to that of particle-type BCP, which has been regarded as the gold standard of allografts. In previous studies, combining collagen with BCP enhanced clinical manipulation without impairing bone regeneration. ${ }^{10,14}$

In the present study, applying DOPA for membrane stability increased new bone formation in the late healing period; however, there was no significant difference in bone formation during the early healing period. This might be related to the histologic finding that the infiltration of monocytes was prominent in the early healing period. Increased levels of monocytes elevate pro-inflammatory cytokines such as interleukin-1 and tumor necrosis factor-alpha, and differentiation of osteoclasts. ${ }^{15}$ Thus, increased stability from DOPA application can exhibit its effect after early inflammation diminishes. 
DOPA is an amino acid that is mainly involved in the adhesive mechanism of MAP. ${ }^{8}$ In the present study, MNGCs were observed until the late healing period underneath the collagen membrane where DOPA was applied. The presence of MNGC was regarded as a host-graft material rejection or sign of inflammation; however, recent studies have reported different meanings regarding the presence of MNGC. ${ }^{16}$ Recent studies have reported that the formation of MNGC plays an important role in inflammation-mediated vascularization of collagen matrix. ${ }^{17,18}$ In histological observations, biomaterialinduced MNGC, which are not found in the physiological wound healing process, were observed around the implanted collagen matrix, and peripheral vascularization and disintegration of the collagen matrix occurred due to the enhanced number of MNGC. ${ }^{18}$ However, there is no clear histologic difference between pathologic MNGC, such as those induced by sarcoidosis, and biomaterial-induced MNGC. Therefore it is ambiguous to define the formation of MNGC as either a physiologic or pathologic reaction. ${ }^{17}$ Further investigation is needed to confirm the role and function of MNGC.

There were a few limitations in this study. Since the rabbit calvaria model is flat and immobile, it cannot accurately represent the clinical field, which is prone to movement and pressure. Canine models that more closely represent clinical situations or are comparable to the conventional method groups, such as using titanium pin, could be applied for precise evaluation of clinical efficacies with regards to DOPA application. However, within these limitations, we could confirm the biocompatible outcomes of the collagenated synthetic bone graft attached to the collagen membrane using DOPA.

\section{Conclusion}

In conclusion, within the limitations of the present study, the collagenated synthetic bone graft attached to the collagen membrane using DOPA showed its potential for application in guided bone regeneration. However, MNGC were observed until the late healing period. Further investigation is needed to understand the meaning and role of MNGC in GBR.

\section{References}

1. Cha HS, Kim JW, Hwang JH, Ahn KM. Frequency of bone graft in implant surgery. Maxillofac Plast Reconstr Surg 2016;38:1-4.

2. Wessing B, Lettner S, Zechner W. Guided bone regeneration with collagen membranes and particulate graft materials: a systematic review and meta-analysis. Int J Oral Maxillofac Implants 2018;33:87-100.

3. Wang HL, Boyapati L. "PASS” principles for predictable bone regeneration. ImplantDent 2006;15:8- 
17.

4. Trevisiol L, Nocini PF, Albanese M, Sbarbati A, D'Agostino A. Grafting of large mandibular advancement with a collagen-coated bovine bone (Bio-Oss Collagen) in orthognathic surgery. $\mathrm{J}$ Craniofac Surg 2012;23:1343-8.

5. Lee JT, Cha JK, Kim S, Jung UW, Thoma DS, Jung RE. Lateral onlay grafting using different combinations of soft-type synthetic block grafts and resorbable collagen membranes: An experimental in vivo study. Clin Oral Implants Res 2020;31:303-14.

6. Sun YK, Cha JK, Thoma DS, Yoon SR, Lee JS, Choi SH, et al. Bone Regeneration of Peri-Implant Defects Using a Collagen Membrane as a Carrier for Recombinant Human Bone Morphogenetic Protein-2. Biomed Res Int 2018;2018:5437361.

7. Song WK, Kang JH, Cha JK, Lee JS, Paik JW, Jung UW, et al. Biomimetic characteristics of mussel adhesive protein-loaded collagen membrane in guided bone regeneration of rabbit calvarial defects. J Periodontal Implant Sci 2018;48:305-16.

8. Lee H, Scherer NF, Messersmith PB. Single-molecule mechanics of mussel adhesion. Proc Natl Acad Sci U S A. 2006;103:12999-3003.

9. Hong I, Khalid AW, Pae HC, Cha JK, Lee JS, Paik JW, et al. Distinctive bone regeneration of calvarial defects using biphasic calcium phosphate supplemented ultraviolet-crosslinked collagen membrane. J Periodontal Implan Sci 2020;50:14-27.

10. Javier MM, Wui H, Jung RE, Hämmerle CHF, Benic GI. Influence of blinded wound closure on the volume stability of different GBR materials: an in vitro cone-beam computed tomographic examination. Clin Oral Implan Res 2016;27:258-65.

11. Urban IA, Nagursky H, Lozada JL. Horizontal Ridge Augmentation with a Resorbable Membrane and Particulated Autogenous Bone With or Without Anorganic Bovine Bone-Derived Mineral: A Prospective Case Series in 22 Patients. Int J Oral Maxillofac Implants 2011;26:404-14.

12. Song MJ, Amirian J, Linh NTB, Lee BT. Bone morphogenetic protein-2 immobilization on porous PCL-BCP-Col composite scaffolds for bone tissue engineering. J Appl Polym Sci 2017;134:45186.

13. Pae HC, Kang JH, Cha JK, Lee JS, Paik JW, Jung UW, et al. 3D-printed polycaprolactone scaffold mixed with beta-tricalcium phosphate as a bone regenerative material in rabbit calvarial defects. $\mathrm{J}$ Biomed Mater Res B Appl Biomater 2019;107:1254-63.

14. Cha JK, Joo MJ, Yoon S, Lee JS, Choi SH, Jung UW. Sequential healing of onlay bone grafts using combining biomaterials with cross-linked collagen in dogs. Clin Oral Implants Res 2017;28:76-85.

15. Giannobile WV. Host-response therapeutics for periodontal diseases. J Periodontol 2008;79:1592600.

16. Miron RJ, Zohdi H, Fujioka-Kobayashi M, Bosshardt DD. Giant cells around bone biomaterials: Osteoclasts or multi-nucleated giant cells? Acta Biomater 2016;46:15-28.

17. Al-Maawi S, Vorakulpipat C, Orlowska A, Zrnc TA, Sader RA, Kirkpatrick CJ, et al. In vivo Implantation of a Bovine-Derived Collagen Membrane Leads to Changes in the Physiological Cellular Pattern of Wound Healing by the Induction of Multinucleated Giant Cells: An Adverse Reaction? Front Bioeng Biotechnol 2018;6:104.

18. Tanneberger AM, Al-Maawi S, Herrera-Vizcaino C, Orlowska A, Kubesch A, Sader R et al. Multinucleated giant cells within the in vivo implantation bed of a collagen-based biomaterial determine its degradation pattern. Clin Oral Investig 2020;25:859-73. 